The Journal of Vitaminology 5, 161--177 (1959)

\title{
BIOSYNTHESIS OF CAROTENOIDS BY FUNGI
}

\author{
YASUO MASE \\ Takanine Laboratory, Sankyo Co. Ltd., Osaki, Tokyo
}

(Received June 3, 1959)

The occurrence of carotenoids in species of Penicillia has never been observed with the only one exception. Luteraan and Choay (1) mentioned an unspecified Penicillium as a carotene producer but full details of this investigation are not yet available.

The author conducted a survey of carotenogenic activity of a number of fungus strains, and found Penicillium sclerotiorum to produce a considerable amount of carotenoid pigments. In addition to this, Pen. multicolor, Pen. lapidosum and Rhizoctonia zeae have also been found to be carotene producers. The persent report describes the occurrence and identification of the major carotenoid pigments in the above four strains, some culture conditions affecting the carotene formation of Pen. sclerotiorum, and discussion on the biosynthetic pathway of the carotenoid.

\section{EXPERIMENTAL}

\section{Culture of Fungi and Determination of Carotene Content}

Pen. sclerotiorum, Pen. multicolor, Pen. lapidosum, and Rhiz. zeae were grown at $25^{\circ}$ in a liquid medium (designated Medium No. 1) which contained the following ingredients: Difco malt extract, $15 \mathrm{~g}$; maltose, $12.5 \mathrm{~g}$; soluble starch, $2.5 \mathrm{~g}$; glycerol, $2.5 \mathrm{~g}$; dibasic potassium phosphate, $1 \mathrm{~g}$; ammonium chloride, $1 \mathrm{~g}$; Difco peptone, $1 \mathrm{~g}$; distilled water to make $1000 \mathrm{ml}$. The $\mathrm{pH}$ of the medium was adjusted to approximately 5 with $10 \mathrm{~N}$ sulfuric acid and then sterilized at $120^{\circ}$ for $15 \mathrm{~min}$. Cultures were grown in 500-ml Erlenmeyer flasks containing $100 \mathrm{ml}$ of the medium. After a suitable period of growth the mycelial mats were harvested on a Buchner funnel, washed with distilled water, and extracted in a Waring blendor with acetone and hexane $(100: 75$, $\mathrm{v} / \mathrm{v})$. The mycelial mat was filtered and extracted twice more with 200-ml portions of acetone. The filtrates were combined in a separatory funnel and treated with $250 \mathrm{ml}$ of water. The aqueous hypophase was discarded, and the hexane epiphase was washed three times with the mixture containing $300-\mathrm{ml}$ portions of water and one $50-\mathrm{ml}$ portion of $90 \%$ aqueous methanol. The methanol extract contained a colored substance of undetermined nature.

The hexane epiphase solution was extracted with $50 \mathrm{ml}$ of methanolic potassium hydroxide solution (methanol-water-potassium hydroxide, $90: 10: 20$, $\mathrm{v} / \mathrm{v} / \mathrm{w}$ ), and then with another $50 \mathrm{ml}$ of $90 \%$ methanol. This final extract 
was colorless. The epiphase was washed four times with equal volumes of water, and finally filtered through a layer of anhydrous sodium sulfate. The methanolic extracts and washings were discarded. The hexane extract was made to $100 \mathrm{ml}$ in a volumetric flask.

The absorbance of the hexane solution was determined at $450 \mathrm{~m} \mu$ with a Beckman DU spectrophotometer. Total carotene, calculated as $\beta$-carotene, was determined from the following formula:

$$
\mu \mathrm{g} \text { carotene }=\frac{\text { absorbance }}{258} \times 1000 \times 100
$$

To determine the weight of the dry mycelia, a duplicate mycelial mat of each culture was heated at $100^{\circ}$ for $15 \mathrm{~min}$, filtered on a Buchner funnel, washed three times with distilled water, dried at $105-110^{\circ}$ for $4.5 \mathrm{hr}$ and weighed.

\section{Identification of the Pigment}

The strains were cultured for 15 days at $25^{\circ}$ in thirty $500-\mathrm{ml}$ Erenmeyer flasks containing $100 \mathrm{ml}$ of the medium No. 1 . The mycelial mats were collected, and the carotenes were extracted in dry hexane solution by the procedure shown elsewhere (2). Preliminary separations were made on magnesiasupercel (1:1) and complete separation on lime. All major zones were removed from the developed column by sectioning, eluted with ethyl ether, concentrated to dryness, and redissolved in hexane or isooctane. Complete absorption spectra were obtained in the range 520 to $275 \mathrm{~m} \mu$. These pigments were identified from the chromatographic behaviors and absorption spectra.

\section{Environmental Effects on Carotenogenesis}

Pen. sclerotiorum was grown under various environmental conditions on the medium No. 1 or chemically defined media (designated No. 2), with some modification occasionally, which contained the following basal ingredients: Glucose, $40 \mathrm{~g}$; citric acid, $10 \mathrm{~g}$; $\left(\mathrm{NH}_{4}\right)_{2} \mathrm{SO}_{4}, 1.5 \mathrm{~g} ; \mathrm{KH}_{2} \mathrm{PO}_{4}, 0.5 \mathrm{~g} ; \mathrm{MgCl}_{2} \cdot 6 \mathrm{H}_{2} \mathrm{O}$, $0.5 \mathrm{~g} ; \quad \mathrm{B}\left(\right.$ as $\left.\mathrm{Na}_{2} \mathrm{~B}_{4} \mathrm{O}_{7} \cdot 10 \mathrm{H}_{2} \mathrm{O}\right), 10 \mu \mathrm{g} ; \quad$ Mo (as $\left(\mathrm{NH}_{4}\right)_{6} \mathrm{Mo}_{7} \mathrm{O}_{27} \cdot 4 \mathrm{H}_{2} \mathrm{O}$ ), $20 \mu \mathrm{g} ; \mathrm{Zn}$ (as $\mathrm{ZnSO}_{4} \cdot 7 \mathrm{H}_{2} \mathrm{O}$ ), $200 \mu \mathrm{g}$; $\mathrm{Cu}$ (as $\mathrm{CuCl}_{2} \cdot 2 \mathrm{H}_{2} \mathrm{O}$ ), $100 \mu \mathrm{g}$; $\mathrm{Mn}$ (as $\mathrm{MnCl}_{2} \cdot 4 \mathrm{H}_{2} \mathrm{O}$ ), $20 \mu \mathrm{g}$; $\mathrm{Fe}\left(\right.$ as $\left.\mathrm{FeSO}_{4} \cdot 7 \mathrm{H}_{2} \mathrm{O}\right), 250 \mu \mathrm{g}$; $\mathrm{Co}\left(\right.$ as $\left.\mathrm{CoCl}_{2} \cdot 6 \mathrm{H}_{2} \mathrm{O}\right), 5 \mu \mathrm{g}$; distilled water to make $1000 \mathrm{ml}$.

Effects of various culture conditions on its growth and carotene formation were investigated in some detail. Extraction of pigments and determination of total carotene were conducted as shown previously.

\section{RESULTS AND DISCUSSION}

\section{Survey}

A survey of carotenogenic activity of 72 fungus strains were conducted. In addition to the strains known as carotene producers, Pen. sclerotiorum, Pen. multicolor, Pen.lapidosum, and Rhizoctonia zeae were found to produce carotene when grown on Difco wort agar media. Table I shows the growth and total carotene content of the new carotene producers, and the carotenoids have been shown to be absent from the fungi listed in Table II.

Among the tested strains, Pen. sclerotiorum NRRL 2074 was found to be 
TABLE I

Growth and Total Carotene Content on Wort Agar (at 25 $\left.{ }^{\circ}\right)$

\begin{tabular}{|c|c|c|c|c|}
\hline Strain & NRRL No. & $\begin{array}{l}\text { Duration of } \\
\text { incubation }\end{array}$ & $\begin{array}{l}\text { Dry weight } \\
\text { per } 100 \mathrm{~m} 1 \\
\text { medium }\end{array}$ & $\begin{array}{l}\text { Total } \\
\text { carotene }\end{array}$ \\
\hline & & days & $m g$ & ug per $g$ dry \\
\hline Pen. sclerotiorum & 2074 & 14 & 773 & 696 \\
\hline " & 2076 & 14 & 792 & 9 \\
\hline Pen. multicolor & 764 & 15 & 947 & 13 \\
\hline " & 2058 & 15 & 848 & 12 \\
\hline$"$ & 2059 & 15 & 790 & 25 \\
\hline$"$ & 2060 & 15 & 750 & 8 \\
\hline$"$ & $A-2324$ & 15 & 1107 & 9 \\
\hline Pen. lapidosum & 718 & 21 & 897 & 32 \\
\hline " & 2084 & 16 & 968 & 14 \\
\hline Rhizoctonia zeae & - & 12 & 556 & 88 \\
\hline
\end{tabular}

TABLE II

Fungi in Which Carotenoids Have Not Been Shown to Be Present

\begin{tabular}{|c|c|c|c|c|}
\hline Penicillium & asperum & Penicillium & parvum & Aspergillus chevalieri \\
\hline$"$ & avellaneum & $"$ & purpurogenum & citrosporus \\
\hline$"$ & baarnense & $"$ & pusillum & flavus \\
\hline$"$ & chrysogenum & , & roquefort $i$ & ochraceus \\
\hline ", & duclauxi & $"$ & rubrum & oryzae \\
\hline ", & funiculosum & ", & stipitatum & tamarii \\
\hline ", & gladioli & ", & striatum & ustus \\
\hline$"$ & implicatum & ", & substeritium & Gibberella zeae \\
\hline ", & janthinellum & $"$ & thomii & Monascus purprea \\
\hline$"$ & javanicum & $"$ & turbatum & Rhizopus delemar \\
\hline$"$ & luteum & $"$ & wortmanii & Trichoderma liquerum \\
\hline$"$ & notatum & & & \\
\hline
\end{tabular}

the most promising carotene producer. This species, belonging to the Penicillium thomii series, was first isolated in Java in 1935 (3) and has been maintained in the NRRL collection as strain NRRL 2074. This species has been represented also by Pen. sclerotiorum NRRL 2076 which was isolated from soil collected in Brazil (4).

Pen. sclerotiorum has been of special interest because of the brilliant orangered color of the mycelia which characteristically surround the sclerotia of this species when cultured on Czapek-Dox medium. In 1940 Curtin and Reilly (5) isolated an acidic pigment from Pen. sclerotiorum in a yield comprising $2 \%$ of the dry mycelial mat. This acidic pigment, which was isolated also from Pen. multicolor $(6,7)$, was named sclerotiorine. It is extractable from hexane with alkali. Hence it is easily distinguished from carotene by this property. Characteristically media such as Czapek-Dox agar, which favor the production of copious quantities of sclerotiorine, inhibited the formation of carotenes. The inverse effect of different media on the formation of both sclerotiorine and carotene suggests that possibly sclerotiorine is a precursor for carotene or a metabolic product of carotenogenesis in culture 2074.

From the analysis of sclerotiorine and its derivatives, the most probable 
empirical formula was shown to be $\mathrm{C}_{21} \mathrm{H}_{23} \mathrm{O}_{5} \mathrm{Cl}(6,7)$. When sclerotiorine was warmed with alkali, it was hydrolyzed to a crystaline monobasic acid containing conjugated double bonds, 4,6-dimethyl-octa-2,4-dienoic acid. The isoprenoic nature of sclerotiorine and of the carotenes lends support to the possibility that they have a metabolic interrelationship.

Culture 2074 produced no extracellular carotene in the agar or liquid portion of the media, but the color of the mycelial mats was greater between the mats and the culture media.

\section{Identification of Pigments}

A preliminary separation of extracted total carotene was made on magnesia-Supercel, and a complete separation was made on lime. Each major zone was removed from the chromatograms and their properties are summarized in

TABLE III

Wavelength of Maximum Absorption of Various Carotenoids Produced by Pen. sclerotiorum (in Hexane)

\begin{tabular}{|c|c|c|c|c|c|c|c|c|c|c|}
\hline \multirow{3}{*}{ 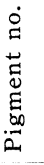 } & \multirow{3}{*}{ Carotene indicated } & \multicolumn{8}{|c|}{ Maxima } & \multirow{3}{*}{$\begin{array}{l}\text { Per cent } \\
\text { of total } \\
\text { pigment }\end{array}$} \\
\hline & & \multicolumn{2}{|c|}{1} & \multicolumn{2}{|r|}{2} & \multicolumn{2}{|c|}{3} & \multicolumn{2}{|c|}{ Cis peak } & \\
\hline & & $\mathrm{m} / \mu$ & $\mathrm{A}^{a}$ & $\mathrm{~m} \mu$ & A & $\mathrm{m} \mu$ & A & $\mathrm{m}_{i}$ & A & \\
\hline 1 & $\alpha$-Caroten & 475 & 0.860 & 445 & $1.000^{b}$ & 422 & 0.718 & 331 & 0.210 & 0.2 \\
\hline 2 & Neo- $\beta$-carotene-1 & 470 & 0.801 & 447 & 1.000 & & & 336 & 0.561 & 1.5 \\
\hline 3 & Neo- $\beta$-carotene- 2 & 472 & 0.832 & 445 & 1.000 & & & 338 & 0.385 & 2.9 \\
\hline 4 & $\beta$-Carotene & 478 & 0.902 & 450 & 1.000 & & & 335 & 0.050 & 64.6 \\
\hline 5 & Neo- $\beta$-carotene -3 & 474 & 0.875 & 445 & 1.000 & & & 325 & 0.135 & 1.2 \\
\hline 6 & Zeacarotene-1 & 452 & 0.841 & 426 & 1.000 & 406 & 0.715 & & & 0.3 \\
\hline 7 & Zeacarotene--2 & 454 & 0.854 & 427 & 1.000 & 404 & 0.712 & 286 & 1.190 & 0.4 \\
\hline 8 & Neo- $\gamma$-carotene-1 & 486 & 0.781 & 456 & 1.000 & 434 & 0.764 & 347 & 0.329 & 1.1 \\
\hline 9 & Neo- $\gamma$-carotene- 2 & 486 & 0.801 & 456 & 1.000 & 432 & 0.734 & 348 & 0.271 & 1.7 \\
\hline 10 & $\gamma$-Carotene & 492 & 0.862 & 461 & 1.000 & 436 & 0.710 & 350 & 0.063 & 25.8 \\
\hline 11 & Unknown & 517 & 0.716 & 483 & 1.000 & 456 & 0.905 & & & 0.3 \\
\hline
\end{tabular}

${ }^{a} \mathrm{~A}=\log I_{0} / I$.

${ }^{b}$ The highest absorption maximum of each carotenoid was arbitrarily given a value of 1.000 .

The same are applicable in the following Tables.

TABLE IV

Wavelength of Maximum Absorption of Various Carotenoids Produced by Pen. multicolor (in Hexane)

\begin{tabular}{|c|c|c|c|c|c|c|c|c|}
\hline \multirow{3}{*}{$\begin{array}{l}\text { Pigment } \\
\text { no. }\end{array}$} & \multirow{3}{*}{$\begin{array}{l}\text { Carotene } \\
\text { indicated }\end{array}$} & \multicolumn{6}{|c|}{ Maxima } & \multirow{3}{*}{$\begin{array}{l}\text { Per cent } \\
\text { of total } \\
\text { pigment }\end{array}$} \\
\hline & & \multicolumn{2}{|c|}{1} & \multicolumn{2}{|c|}{2} & \multicolumn{2}{|c|}{3} & \\
\hline & & $\mathrm{m} \mu$ & A & $\mathrm{m} \mu$ & A & $\mathrm{m} \mu$ & A & \\
\hline 1 & $\beta$-Carotene & 478 & 0.902 & 450 & 1.000 & & & 17.9 \\
\hline 2 & Zeacarotene & 452 & 0.841 & 426 & 1.000 & 406 & 0.715 & 1.2 \\
\hline 3 & $\boldsymbol{\gamma}$-Carotene & 492 & 0.862 & 461 & 1.000 & 436 & 0.710 & 33.0 \\
\hline 4 & Neurosporene & 468.5 & 0.975 & 439.5 & 1.000 & 416 & 0.689 & 3.4 \\
\hline
\end{tabular}


Tables III-VI.

TABLE V

Wavelength of Maximum Absorption of Various Carotenoids Produced by Pen. lapidosum (in Hexane)

\begin{tabular}{|c|c|c|c|c|c|c|c|c|c|c|}
\hline \multirow{3}{*}{$\begin{array}{l}\dot{0} \\
\dot{a} \\
\vec{E} \\
\stackrel{\infty}{E} \\
\stackrel{0}{a}\end{array}$} & \multirow{3}{*}{$\begin{array}{l}\text { Carotene } \\
\text { indicated }\end{array}$} & \multicolumn{8}{|c|}{ Maxima } & \multirow{3}{*}{$\begin{array}{l}\text { Per cent } \\
\text { of total } \\
\text { pigment }\end{array}$} \\
\hline & & \multicolumn{2}{|c|}{1} & \multicolumn{2}{|c|}{2} & \multicolumn{2}{|r|}{3} & \multicolumn{2}{|r|}{4} & \\
\hline & & $\mathrm{m} \mu$ & A & $\mathrm{m} \mu$ & A & $\mathrm{m}_{\mu}$ & $\mathrm{A}$ & $\mathrm{m} / \mu$ & A & \\
\hline 1 & Ergosterol & 293.5 & 0.573 & 282 & 1.000 & 271 & 0.945 & 262 & 0.671 & - \\
\hline 2 & $\boldsymbol{\gamma}$-Carotene & 492 & 0.843 & 461 & 1.000 & 436 & 0.714 & & & 17.2 \\
\hline 3 & Pigment L & 504.5 & 0.799 & 474.5 & 1.000 & 360 & 0.145 & & & 43.4 \\
\hline
\end{tabular}

TABLE VI

Wavelength of Maximum Absorption of Various Carotenoids

Produced by Rhizoctonia zeae

\begin{tabular}{|c|c|c|c|c|c|c|c|c|c|}
\hline \multirow{3}{*}{ 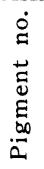 } & \multirow{3}{*}{$\begin{array}{l}\text { Carotene } \\
\text { indicated }\end{array}$} & \multirow{3}{*}{ Solvent } & \multicolumn{6}{|c|}{ Maxima } & \multirow{3}{*}{$\begin{array}{l}\text { Per cent } \\
\text { of total } \\
\text { pigment }\end{array}$} \\
\hline & & & \multicolumn{2}{|c|}{1} & \multicolumn{2}{|c|}{2} & \multicolumn{2}{|r|}{3} & \\
\hline & & & $\mathrm{m} \mu$ & $\mathrm{A}$ & $\mathrm{m} \mu$ & A & $\mathrm{m} / \mu$ & A & \\
\hline 1 & Phytofluene & Isooctane & 367 & 0.568 & 348 & 1.000 & 332 & 0.920 & 2.1 \\
\hline 2 & $\beta$-Carotene & Hexane & 478 & 0.898 & 450 & 1.000 & 335 & 0.065 & 33.4 \\
\hline 3 & $\boldsymbol{\gamma}$-Carotene & Hexane & 491.5 & 0.856 & 460.5 & 1.000 & 436 & 0.721 & 5.8 \\
\hline
\end{tabular}

Pen. sclerotiorum NRRL 2074 produced about $1 \mathrm{mg}$ of total carotene per $\mathrm{g}$ of mycelium dry weight, of which approximately $65 \%$ was $\beta$-carotene and approximately $25 \%$ was $\gamma$-carotene. . In addition, as minor pigments, $\alpha$-carotene, 3 -cis-isomers of $\beta$-carotene, 2 -cis-isomers of $\gamma$-carotene, and zeacarotene were identified.

Pen. multicolor NRRL 2059 produced about $50 \mu \mathrm{g}$ of total carotene per gram of mycelium dry weight, of which ca. $18 \%$ was $\beta$-carotene, ca. $33 \% \gamma$-carotene, ca. $3 \%$ neurosporene, and ca. $1 \%$ zeacarotene.

Pen. lapidosum NRRL 718 produced about $70 \mu \mathrm{g}$ of total carotene per $\mathrm{g}$ of mycelium dry weight, of which ca. $43 \%$ was the pigment L (unidentified), and ca. $17 \% \gamma$-carotene. In addition, ergosterol was identified.

Rhizoctonia zeae produced about $90 \mu \mathrm{g}$ of total carotene per $\mathrm{g}$ of mycelium dry weight, of which ca. $33 \%$ was $\beta$-carotene, ca. $6 \% \gamma$-carotene, and ca. $2 \%$ phytofluene.

\section{Environmental Effects on Carotenogenesis}

pH of Medium - Pen. sclerotiorum NRRL 2074 was cultured on several media. As shown in Fig. 1 and Tables VII-IX for instance, in all cases showed the minimum value in 6 to 9 days after inoculation and thereafter increased gradually. Its carotene production seemed to be markedly affected 


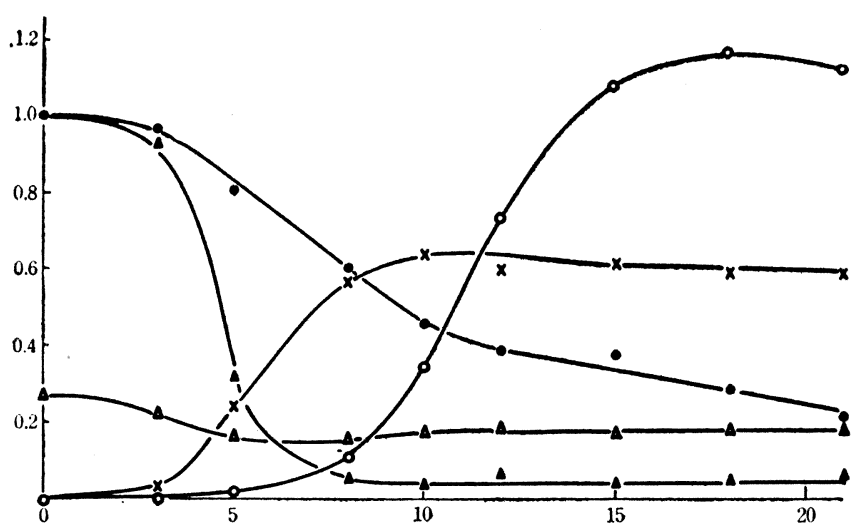

Time, days

FIg. 1 Time Course Data during Incubation of Penicillium sclerotiorum

Mycelium dry weight, $\mathrm{g} / 100 \mathrm{ml}$ medium

Total carotene, $\mathrm{mg} / \mathrm{g}$ dry mycelium .

$\triangle \mathrm{pH}$ of medium

- Residual sugar (initial content $=1.0$ )

$\Delta \quad$ Residual nitrogen (initial content $=1.0$ ) by the $\mathrm{pH}$ value of the medium after it attained to the minimum value. The optimum $\mathrm{pH}$ for its carotenogenesis was found to be $2.00 \pm 0.25$.

The total carotene produced on an acidic medium (initial $\mathrm{pH}:$ 2.31) was about twice as much as that on neutral media (initial $\mathrm{pH}: 4.59$ and 6.98). On the neutral media xanthophylls and acidic
TABLE VII

Effect of $p H$ (1) $\left(25^{\circ}, 15\right.$ days $)$

\begin{tabular}{|c|c|c|c|}
\hline \multicolumn{2}{|c|}{$\mathrm{pH}$ of medium } & \multirow{2}{*}{$\begin{array}{l}\text { Yield of } \\
\text { dry matter }\end{array}$} & \multirow{2}{*}{$\begin{array}{l}\text { Total } \\
\text { carotene }\end{array}$} \\
\hline Initial & Final & & \\
\hline & & $\mathrm{mg} / 100 \mathrm{ml}$ & $\mu g / g d r y$ \\
\hline 1.03 & 1.00 & 0 & - \\
\hline 1.58 & 1.39 & 541 & 43 \\
\hline 2.10 & 1.59 & 549 & 268 \\
\hline 2.61 & 1.71 & 643 & 436 \\
\hline 3.11 & 1.80 & 690 & 536 \\
\hline 4.11 & 1.82 & 640 & 734 \\
\hline 5.00 & 1.88 & 710 & 689 \\
\hline 5.80 & 1.90 & 651 & 784 \\
\hline 6.58 & 2.00 & 658 & 801 \\
\hline 7.08 & 2.19 & 685 & 935 \\
\hline 7.90 & 2.80 & 706 & 734 \\
\hline 8.15 & 4.60 & 747 & 234 \\
\hline
\end{tabular}

TABLE VIII

Effect of $p H$ (2) $\left(25^{\circ}, 15\right.$ days $)$

\begin{tabular}{|c|c|c|c|}
\hline \multicolumn{2}{|c|}{$\mathrm{pH}$ of medium } & \multirow{2}{*}{$\begin{array}{l}\text { Yield of dry } \\
\text { matter }\end{array}$} & \multirow{2}{*}{$\begin{array}{l}\text { Total } \\
\text { carotene }\end{array}$} \\
\hline Initial & Final & & \\
\hline & & $\mathrm{mg} / 100 \mathrm{ml}$ & $\mu g / g$ dry \\
\hline 1.70 & 1.36 & 622 & 201 \\
\hline 1.93 & 1.57 & 664 & 318 \\
\hline 2.19 & 1.70 & 734 & 462 \\
\hline 2.43 & 1.86 & 722 & 725 \\
\hline 2.69 & 2.09 & 784 & 1227 \\
\hline 3.15 & 6.31 & 806 & 765 \\
\hline 4.15 & 8.02 & 884 & 298 \\
\hline 5.10 & 7.89 & 870 & 336 \\
\hline
\end{tabular}

pigments were produced, but not on the acidic medium. Although the amounts of $\beta$-carotene and zeacarotene

produced on those three media did not much vary, the amount of $r$-carotene produced on the acidic medium was much greater than the amounts on the neutral media. Production of $\gamma$-carotene appeared to be accelerated on the acidic medium.

General Conditions - Growth of Pen. sclerotiorum was observed at temperatures between 12 and $32^{\circ}$, but maximum carotene production was attained at $24^{\circ}$. The data are shown in Fig. 2. The optimum depth of the medium for its carotenogenesis seemed to be approximately $1 \mathrm{~cm}$. The smaller the medium concentration, the greater was the total carotene per $g$ dry mat. 
TABLE IX

Effect of $p H(3)\left(25^{\circ}, 15\right.$ days $)$

\begin{tabular}{cccc}
\hline \multicolumn{2}{c}{$\mathrm{pH}$ of medium } & $\begin{array}{c}\text { Yield of } \\
\text { dry matter }\end{array}$ & $\begin{array}{l}\text { Total } \\
\text { carotene }\end{array}$ \\
\cline { 1 - 2 } Initial & Final & & \\
\hline & & $m g / \mathbf{1 0 0} \boldsymbol{m l}$ & $\mu \mathrm{g} / \mathrm{g}$ dry \\
1.35 & 1.20 & 0 & $-\overline{7}$ \\
1.75 & 1.39 & 344 & 247 \\
1.95 & 1.59 & 372 & 394 \\
2.30 & 1.75 & 454 & 505 \\
2.60 & 1.95 & 524 & 331 \\
3.40 & 2.90 & 678 & 86 \\
4.48 & 4.05 & 714 & 54 \\
6.20 & 4.85 & 736 & 77 \\
7.10 & 4.92 & 525 & 77 \\
\hline
\end{tabular}

Even after maximum growth was attained and nitrogen assimilation was ceased, consumption of sugar was further continued, which seemed

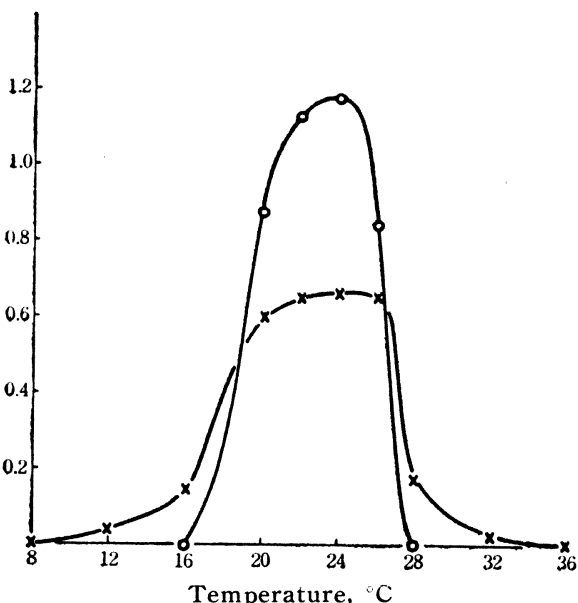

FIt. 2 Effect of Temperature on the Carotenogenesis of Pen. sclerotiorum

Mycelium dry weight, g/100 ml medium Total carotene, $\mathrm{mg} / \mathrm{g}$ dry mycelium to be used for carotene formation.

The time course data during incubation was shown in Fig. 1.

The optimum light intensity was about 1 foot candle for 15 day cultivation. Stronger light seemed to inhibit carotene formation or to stimulate

TABLE X

Effect of Carbon Sources $\left(25^{\circ}\right)$

\begin{tabular}{|c|c|c|c|c|c|c|}
\hline \multirow[b]{2}{*}{ Carbon source } & \multicolumn{3}{|c|}{15 days } & \multicolumn{3}{|c|}{20 days } \\
\hline & $\begin{array}{l}\text { Final } \\
\mathrm{pH}\end{array}$ & $\begin{array}{l}\text { Yield of } \\
\text { dry matter }\end{array}$ & $\begin{array}{l}\text { Total } \\
\text { carotene }\end{array}$ & $\begin{array}{l}\text { Final } \\
\mathrm{pH}\end{array}$ & $\begin{array}{l}\text { Yield of } \\
\text { dry matter }\end{array}$ & $\begin{array}{l}\text { Total } \\
\text { carotene }\end{array}$ \\
\hline & & $m g / 100 m i l$ & $\mu g / g$ dry & & $\mathrm{mg} / 100 \mathrm{ml}$ & $\mu g / g d r y$ \\
\hline Acetic acid & 2.30 & 0 & 0 & 2.30 & 0 & 0 \\
\hline $\mathrm{L}(+)$-Arabinose & 1.75 & 548 & 1242 & 1.76 & 529 & 1440 \\
\hline $\mathrm{D}(+)$-Cellobiose & 1.80 & 594 & 950 & 1.82 & 581 & 1370 \\
\hline Citric acid & 2.25 & 631 & 756 & 3.80 & 547 & 850 \\
\hline Dextrin & 1.74 & 543 & 568 & 1.75 & 546 & 320 \\
\hline Diglycerol & 1.82 & 247 & 548 & 1.80 & 236 & 284 \\
\hline Ethyl alcohol & 2.30 & 0 & 0 & 2.30 & 0 & 0 \\
\hline $\mathrm{D}(-)$-Fructose & 1.72 & 672 & 440 & 1.70 & 673 & 595 \\
\hline $\mathrm{D}(+)$-Galactose & 1.80 & 730 & 630 & 1.83 & 751 & 690 \\
\hline D-Glucose & 1.77 & 633 & 959 & 1.81 & 620 & 886 \\
\hline Glycerol & 1.70 & 577 & 981 & 1.69 & 605 & 990 \\
\hline Lactose & 2.02 & 508 & 386 & 2.04 & 424 & 716 \\
\hline $\mathrm{D}(+)$-Maltose & 1.82 & 567 & 441 & 1.81 & 629 & 586 \\
\hline Mannitol & 1.70 & 526 & 671 & 1.73 & 521 & 524 \\
\hline Pectin & 3.00 & 620 & 431 & 3.25 & 608 & 174 \\
\hline $\mathrm{D}(+)$-Raffinose & 1.82 & 635 & 598 & 1.90 & 640 & 804 \\
\hline D-Ribose & 1.79 & 634 & 1193 & 1.84 & 629 & 1096 \\
\hline Sorbitol & 1.70 & 492 & 736 & 1.65 & 525 & 707 \\
\hline Soluble starch & 1.75 & 555 & 673 & 1.70 & 567 & 655 \\
\hline Succinic acid & 1.81 & 515 & 476 & 1.75 & 654 & 897 \\
\hline Saccharose & 1.74 & 625 & 726 & 1.80 & 617 & 738 \\
\hline $\mathrm{D}(+)-\mathrm{Xyl}$ lose & 1.80 & 656 & 1173 & 1.80 & 678 & 1318 \\
\hline
\end{tabular}


carotene destruction, while weaker light appeared to stimulate the formation of xanthophylls and acidic pigments. Direct contact with the air seemed to be necessary for the carotene formation.

The suitable inoculum size was found to be approximately 1600 spores per ml medium.

Carbon and Nitrogen Sources - It is conceivable that if nitrogen is in excess the most part of carbon is used up in protein synthesis, leaving scarcely

TABLE XI

Effect of Nitrogen Sources $\left(25^{\circ}\right)$

\begin{tabular}{|c|c|c|c|c|c|c|}
\hline \multirow[b]{2}{*}{ Nitrogen source } & \multicolumn{3}{|c|}{12 days } & \multicolumn{3}{|c|}{20 days } \\
\hline & $\begin{array}{l}\text { Final } \\
\mathrm{pH}\end{array}$ & $\begin{array}{l}\text { Yield of } \\
\text { dry matter }\end{array}$ & $\begin{array}{l}\text { Total } \\
\text { carotene }\end{array}$ & $\begin{array}{l}\text { Final } \\
\mathrm{pH}\end{array}$ & $\begin{array}{l}\text { Yield of } \\
\text { dry matter }\end{array}$ & $\begin{array}{l}\text { Total } \\
\text { carotene }\end{array}$ \\
\hline & & $\mathrm{mg} / 100 \mathrm{ml}$ & $\mu g / g d r y$ & & $m g / 100 m l$ & $\mu g / g d r y$ \\
\hline $\mathrm{NH}_{4} \mathrm{Cl}$ & 1.76 & 760 & 237 & 1.78 & 785 & 181 \\
\hline $\mathrm{NH}_{4} \mathrm{NO}_{3}$ & 3.82 & 827 & 335 & 3.80 & 888 & 153 \\
\hline$\left(\mathrm{NH}_{4}\right)_{2} \mathrm{HPO}_{4}$ & 1.95 & 693 & 530 & 1.98 & 750 & 445 \\
\hline$\left(\mathrm{NH}_{4}\right)_{2} \mathrm{SO}_{4}$ & 1.72 & 573 & 708 & 1.80 & 687 & 772 \\
\hline DL- $\boldsymbol{\alpha}$-Alanine & 2.22 & 650 & 423 & 2.30 & 703 & 721 \\
\hline L-Arginine & 2.10 & 391 & 220 & 2.20 & 414 & 404 \\
\hline L-Asparagine & 2.27 & 662 & 754 & 2.50 & 667 & 975 \\
\hline L-Aspartic acid & 2.29 & 688 & 503 & 2.50 & 751 & 715 \\
\hline$\beta$-Alanine & 1.81 & 777 & 676 & 1.70 & 762 & 906 \\
\hline Betaine & 2.00 & 660 & 1370 & 2.00 & 782 & 1850 \\
\hline Casein & 2.25 & 619 & 324 & 2.24 & 656 & 1071 \\
\hline Choline $\cdot \mathrm{C} 1$ & 1.71 & 592 & 1280 & 1.70 & 701 & 1295 \\
\hline $\mathrm{L}$-Cysteine $\cdot \mathrm{HC} 1$ & 1.55 & 500 & 900 & 1.60 & 595 & 1022 \\
\hline L-Cystine & 1.80 & 257 & 983 & 1.62 & 685 & 750 \\
\hline Ethanolamine & 1.80 & 454 & 782 & 1.88 & 687 & 829 \\
\hline $\boldsymbol{\gamma}$-Aminobutyric acid & 1.81 & 807 & 606 & 1.75 & 751 & 878 \\
\hline L-Glutamic acid & 2.28 & 686 & 475 & 2.38 & 772 & 712 \\
\hline Glycine & 2.29 & 654 & 338 & 2.48 & 732 & 726 \\
\hline L-Histidine & 2.02 & 614 & 376 & 2.20 & 676 & 801 \\
\hline DL-Isoleucine & 2.22 & 527 & 696 & 2.40 & 840 & 851 \\
\hline L-Leucine & 2.15 & 652 & 690 & 2.41 & 884 & 914 \\
\hline L-Lysine & 2.15 & 125 & 384 & 2.00 & 506 & 596 \\
\hline DL-Methionine & 2.00 & 472 & 142 & 1.88 & 690 & 537 \\
\hline Peptone & 2.18 & 539 & 475 & 2.19 & 541 & 1291 \\
\hline $\mathrm{KNO}_{3}$ & 5.60 & 898 & 101 & 5.75 & 1188 & 120 \\
\hline $\mathrm{KNO}_{2}$ & 2.30 & 0 & 0 & 2.30 & 0 & 0 \\
\hline L-Phenyla lanine & 2.20 & 601 & 276 & 2.37 & 286 & 748 \\
\hline I-Proline & 2.30 & 682 & 318 & 2.50 & 766 & 602 \\
\hline DL-Serine & 2.26 & 677 & 207 & 2.54 & 859 & 485 \\
\hline Thiourea & 2.30 & 0 & 0 & 2.30 & 0 & 0 \\
\hline DL-Threonine & 2.28 & 586 & 701 & 2.58 & 820 & 686 \\
\hline $\mathrm{L}$-Tryptophan & 2.28 & 748 & 350 & 2.75 & 986 & 717 \\
\hline DL-Tyrosine & 2.20 & 631 & 369 & 2.41 & 548 & 670 \\
\hline Urea & 2.38 & 112 & 250 & 2.35 & 116 & 302 \\
\hline Uric acid & 2.30 & 0 & 0 & 2.30 & 0 & 0 \\
\hline DL-Valine & 2.22 & 537 & 714 & 2.49 & 670 & 890 \\
\hline
\end{tabular}

any carbon available for carotene synthesis, and that, on the other hand, if carbon is in excess, there remain plenty to spare for carotene production. In the carotenogenesis, the controlling factor seems to be the amount of assimilable carbon, the $\mathrm{C}: \mathrm{N}$ ratio having only limited significance. 
Effects of carbon and nitrogen sources were investigated, and the results obtained were shown in Tables X and XI. Arabinose, cellobiose, xylose, ribose, and glycerol were good carbon sources for the carotene formation; generally, pentoses were better than hexoses. Alanine and leucine were able to be carbon sources for the carotenogenesis. Leucine, isoleucine, valine, betaine, choline, cysteine, asparagine, and $\beta$-alanine were found to be good nitrogen sources. It is very interesting that methyl donors, such as betaine and choline, were best nitrogen sources for the carotene formation by Pen. sclerotiorum.

Mineral Elements - Tables XII and XIII show the effects of mineral

TABLE XII

Effect of Mineral Elements (1) $\left(25^{\circ}\right)$

\begin{tabular}{|c|c|c|c|c|c|c|c|}
\hline \multirow[b]{2}{*}{ Element } & \multirow[b]{2}{*}{$\mathrm{g} / l$} & \multicolumn{3}{|c|}{12 days } & \multicolumn{3}{|c|}{20 days } \\
\hline & & $\begin{array}{l}\text { Final } \\
\mathrm{pH}\end{array}$ & $\begin{array}{l}\text { Yield of } \\
\text { dry matter }\end{array}$ & $\begin{array}{l}\text { Total } \\
\text { carotene }\end{array}$ & $\begin{array}{l}\text { Final } \\
\mathrm{pH}\end{array}$ & $\begin{array}{l}\text { Yield of } \\
\text { dry matter }\end{array}$ & $\begin{array}{l}\text { Total } \\
\text { carotene }\end{array}$ \\
\hline & & & $\mathrm{mg} / 100 \mathrm{ml}$ & $\mu g / g d r y$ & & $\mathrm{mg} / 100 \mathrm{ml}$ & $\mu g / g d r y$ \\
\hline $\mathrm{K}$ & $\begin{array}{l}0 \\
0.033 \\
0.065 \\
0.13 \\
0.26 \\
0.52\end{array}$ & $\begin{array}{l}2.30 \\
1.72 \\
1.75 \\
1.75 \\
1.80 \\
1.82\end{array}$ & $\begin{array}{r}39 \\
531 \\
509 \\
495 \\
487 \\
512\end{array}$ & $\begin{array}{r}0 \\
755 \\
748 \\
579 \\
620 \\
570\end{array}$ & $\begin{array}{l}2.25 \\
1.78 \\
1.80 \\
1.82 \\
1.83 \\
1.85\end{array}$ & $\begin{array}{r}52 \\
587 \\
621 \\
598 \\
605 \\
602\end{array}$ & $\begin{array}{r}0 \\
980 \\
1021 \\
995 \\
1058 \\
1006\end{array}$ \\
\hline $\mathrm{Mg}$ & $\begin{array}{l}0 \\
0.03 \\
0.06 \\
0.12 \\
0.24\end{array}$ & $\begin{array}{l}2.30 \\
1.70 \\
1.70 \\
1.70 \\
1.70\end{array}$ & $\begin{array}{r}0 \\
613 \\
566 \\
613 \\
570\end{array}$ & $\begin{array}{r}0 \\
615 \\
748 \\
633 \\
690\end{array}$ & $\begin{array}{l}2.32 \\
1.75 \\
1.77 \\
1.76 \\
1.75\end{array}$ & $\begin{array}{r}0 \\
715 \\
777 \\
680 \\
669\end{array}$ & $\begin{array}{r}0 \\
930 \\
987 \\
1130 \\
958\end{array}$ \\
\hline $\mathrm{Cl}$ & $\begin{array}{l}0 \\
0.044 \\
0.087 \\
0.175 \\
0.35 \\
0.70\end{array}$ & $\begin{array}{l}1.78 \\
1.78 \\
1.80 \\
1.78 \\
1.80 \\
1.79\end{array}$ & $\begin{array}{l}584 \\
645 \\
635 \\
666 \\
655 \\
596\end{array}$ & $\begin{array}{l}595 \\
526 \\
569 \\
564 \\
601 \\
597\end{array}$ & $\begin{array}{l}1.80 \\
1.80 \\
1.80 \\
1.82 \\
1.83 \\
1.80\end{array}$ & $\begin{array}{l}624 \\
658 \\
682 \\
694 \\
677 \\
638\end{array}$ & $\begin{array}{r}1044 \\
976 \\
954 \\
1092 \\
981 \\
990\end{array}$ \\
\hline $\mathrm{PO}_{4}$ & $\begin{array}{l}0 \\
0.088 \\
0.175 \\
0.35 \\
0.70 \\
1.40\end{array}$ & $\begin{array}{l}2.28 \\
1.70 \\
1.70 \\
1.70 \\
1.71 \\
1.80\end{array}$ & $\begin{array}{r}0 \\
572 \\
527 \\
550 \\
562 \\
567\end{array}$ & $\begin{array}{r}0 \\
690 \\
524 \\
572 \\
655 \\
738\end{array}$ & $\begin{array}{l}2.31 \\
1.86 \\
1.82 \\
1.80 \\
1.82 \\
1.84\end{array}$ & $\begin{array}{r}0 \\
600 \\
580 \\
596 \\
628 \\
613\end{array}$ & $\begin{array}{r}0 \\
999 \\
976 \\
940 \\
1062 \\
1038\end{array}$ \\
\hline $\mathrm{SO}_{4}$ & $\begin{array}{l}0 \\
0.273 \\
0.545 \\
1.09 \\
2.18 \\
4.36\end{array}$ & $\begin{array}{l}2.57 \\
1.78 \\
1.79 \\
1.82 \\
1.82 \\
1.88\end{array}$ & $\begin{array}{r}51 \\
656 \\
593 \\
572 \\
618 \\
696\end{array}$ & $\begin{array}{r}0 \\
418 \\
479 \\
609 \\
536 \\
515\end{array}$ & $\begin{array}{l}2.55 \\
1.78 \\
1.82 \\
1.88 \\
1.84 \\
1.89\end{array}$ & $\begin{array}{r}60 \\
663 \\
569 \\
780 \\
760 \\
890\end{array}$ & $\begin{array}{r}0 \\
880 \\
684 \\
1018 \\
972 \\
1046\end{array}$ \\
\hline
\end{tabular}

elements upon the growth and carotenogenesis of Pen. sclerotiorum. It was found that $\mathrm{C}, \mathrm{H}, \mathrm{O}, \mathrm{P}, \mathrm{K}, \mathrm{N}, \mathrm{S}$, and $\mathrm{Mg}$ were essential elements; $\mathrm{Fe}, \mathrm{Zn}, \mathrm{Mn}$, $\mathrm{Ni}$, and $\mathrm{Li}$ were stimulatory ones; and $\mathrm{Al}, \mathrm{B}, \mathrm{Ba}, \mathrm{Ca}, \mathrm{Cl}, \mathrm{Co}, \mathrm{Cu}, \mathrm{F}, \mathrm{I}, \mathrm{Mo}$, 
TABLE XIII

Effect of Mineral Elements (2) $\left(25^{\circ}\right)$

\begin{tabular}{|c|c|c|c|c|c|c|}
\hline & \multicolumn{3}{|c|}{15 days } & \multicolumn{3}{|c|}{20 days } \\
\hline & $\begin{array}{l}\text { Final } \\
\mathrm{pH}\end{array}$ & $\begin{array}{l}\text { Yield of } \\
\text { dry matter }\end{array}$ & $\begin{array}{l}\text { Total } \\
\text { carotene }\end{array}$ & $\begin{array}{l}\text { Final } \\
\mathrm{pH}\end{array}$ & $\begin{array}{l}\text { Yield of } \\
\text { dry matter }\end{array}$ & $\begin{array}{l}\text { Total } \\
\text { carotene }\end{array}$ \\
\hline & & $\mathrm{mg} / 100 \mathrm{ml}$ & $\mu g / g d r y$ & & $\mathrm{mg} / 100 \mathrm{ml}$ & $\mu g / g$ dry \\
\hline$-B$ & 1.75 & 506 & 1360 & 1.76 & 566 & 1520 \\
\hline - Mo & 1.75 & 500 & 1305 & & & \\
\hline$-\mathrm{Zn}$ & 1.75 & 441 & 431 & 1.75 & 617 & 700 \\
\hline$-\mathrm{Cu}$ & 1.75 & 464 & 1425 & - & - & - \\
\hline$-\mathrm{Mn}$ & 1.75 & 507 & 601 & 1.74 & 583 & 1110 \\
\hline$-\mathrm{Fe}$ & 1.75 & 468 & 1150 & 1.75 & 643 & 974 \\
\hline$-\mathrm{Co}_{0}$ & 1.75 & 484 & 1321 & - & - & - \\
\hline - all & 1.85 & 363 & 408 & 1.78 & 478 & 500 \\
\hline+ all & 1.75 & 494 & 1352 & 1.78 & 574 & 1482 \\
\hline$+1 / 10$ all & 1.77 & 452 & 725 & 1.77 & 509 & 807 \\
\hline$+1 / 10 \mathrm{Zn}$ & - & - & - & - & 635 & 865 \\
\hline$+1 / 10 \mathrm{Mn}$ & - & - & - & - & 594 & 1438 \\
\hline$+1 / 10 \mathrm{Fe}$ & - & - & - & - & 659 & 972 \\
\hline
\end{tabular}

$\mathrm{Na}$, and $\mathrm{Sn}$ were unnecessary.

Vitamins - Table XIV shows the additional effects of various vitamins, and Tables XV-XIX show the effects of niacin, $p$-aminobenzoic acid, folic acid, calcium pantothenate, and menadione, respectively. Carotene production of Pen. sclerotiorum was stimulated by $p$-amino benzoic acid, folic acid, niacin, and menadione. Calcium pantothenate stimulated growth but inhibited caro-

TABLE XIV

Effect of Vitamins $\left(25^{\circ}\right)$

\begin{tabular}{|c|c|c|c|c|c|}
\hline \multirow[b]{2}{*}{ Vitamin } & \multirow{2}{*}{$\begin{array}{l}\text { Concentra- } \\
\text { tion }\end{array}$} & \multicolumn{2}{|c|}{15 days } & \multicolumn{2}{|c|}{20 days } \\
\hline & & $\begin{array}{l}\text { Yield of } \\
\text { dry matter }\end{array}$ & $\begin{array}{l}\text { Total } \\
\text { carotene }\end{array}$ & $\begin{array}{l}\text { Yield of } \\
\text { dry matter }\end{array}$ & $\begin{array}{l}\text { Total } \\
\text { carotene }\end{array}$ \\
\hline & $m g / l$ & $m g / 100 \mathrm{ml}$ & $\mu g / g d r y$ & $\mathrm{mg} / 100 \mathrm{ml}$ & $\mu g / g d r y$ \\
\hline None & - & 519 & 682 & 534 & 873 \\
\hline Thiamine & 100 & 524 & 638 & 532 & 889 \\
\hline Riboflavin & 100 & 530 & 664 & 555 & 862 \\
\hline Niacin & 1000 & 691 & 755 & 864 & 1096 \\
\hline PABA & 100 & 352 & 915 & 423 & 1250 \\
\hline Pyridoxine & 100 & 569 & 700 & 588 & 824 \\
\hline Ca-pantothenate & 100 & 608 & 612 & 715 & 636 \\
\hline Inositol & 1000 & 548 & 618 & 581 & 771 \\
\hline Biotin & 1 & 538 & 583 & 554 & 814 \\
\hline Folic acid & 100 & 546 & 976 & 543 & 1195 \\
\hline Choline & 1000 & 838 & 532 & 943 & 859 \\
\hline $\mathrm{B}_{12}$ & 1 & 525 & 674 & 561 & 895 \\
\hline Ascorbic acid & 100 & 566 & 669 & 576 & 867 \\
\hline$\alpha$-Lipoic $\operatorname{acid}^{a}$ & 10 & 540 & 802 & 548 & 988 \\
\hline$\alpha$-Tocopherol ${ }^{a}$ & 100 & 528 & 786 & 556 & 1022 \\
\hline Menadione $^{a}$ & 100 & 0 & - & 0 & - \\
\hline Ethanol & $5 \mathrm{cc}$ & 535 & 701 & 549 & 1044 \\
\hline
\end{tabular}

${ }^{a}$ Added as ethanol solutions, and the media contained $0.5 \%$ ethanol when inoculated. 
TABLE XV

Effect of Niacin $\left(25^{\circ}, 15\right.$ days)

\begin{tabular}{rcc}
$\begin{array}{c}\text { Concentration } \\
\text { of niacin }\end{array}$ & $\begin{array}{c}\text { Yield of } \\
\text { dry matter }\end{array}$ & $\begin{array}{l}\text { Total } \\
\text { carotene }\end{array}$ \\
\hline $\mathrm{mg} / l$ & $\mathrm{mg} / \mathbf{1 0 0 ~} \mathrm{ml}$ & $\mu \mathrm{g} / \mathrm{g}$ dry \\
0 & 493 & 707 \\
10 & 487 & 812 \\
100 & 538 & 769 \\
1,000 & 671 & 895 \\
10,000 & 694 & 1076
\end{tabular}

TABIE XVII

Effect of Folic Acid $\left(25^{\circ}, 15\right.$ days)

\begin{tabular}{|c|c|c|}
\hline Folic acid & $\begin{array}{l}\text { Yield, dry } \\
\text { matter }\end{array}$ & $\begin{array}{l}\text { Total } \\
\text { carotene }\end{array}$ \\
\hline$m g / l$ & $\mathrm{mg} / 100 \mathrm{ml}$ & $\mu g / g d r y$ \\
\hline 0 & 525 & 668 \\
\hline 1 & 531 & 673 \\
\hline 10 & 529 & 789 \\
\hline 100 & 536 & 970 \\
\hline 1,000 & 548 & 882 \\
\hline
\end{tabular}

tene production.

Organic Acids - Table XX shows the effects of organic acids used as a sole carbon source, and Table XXI the effects of organic acids added to sugar-containing media. Succinic and fumaric acids were good carbon sources for the carotenogenesis of Pen. sclerotiorum. Mevalonic, dimethylacrylic, levulinic, kojic, and fumaric acids stimulated its carotene production. Formic, acetic, propionic, and butyric acids inhibited its growth.

Other Compounds - Table XXII shows the effects of several possible precursors on the carotenogenesis. $\alpha-, \beta-$, and $\psi$-ionones, phytol, isophytol, methylheptenone, squalene, and vitamin A stimulated the carotene production to some extent; while citral, citronellal, geraniol, or isoprene failed to show such an effect.

\section{Spectral and Stereochemical Properties of Carotenes}

In order to study the biosynthesis of the carotenoid belonging to a conjugated polyene, it seems to be one of the most important problems to investigate 
TABLE XX

Effect of Organic Acids Used as Sole Carbon Source $\left(25^{\circ}, 15\right.$ days)

$\begin{array}{lccr}\begin{array}{l}\text { Organic acid } \\ \text { or sugar }\end{array} & \begin{array}{l}\text { Final } \\ \text { pH }\end{array} & \begin{array}{l}\text { Yield of } \\ \text { dry matter }\end{array} & \begin{array}{l}\text { Total } \\ \text { carotene }\end{array} \\ & & m g / \mathbf{1 0 0} \boldsymbol{m l} & \mu \mathrm{\mu} / \mathbf{g} \text { dry } \\ \text { Acetic } & 2.30 & 0 & - \\ \text { Aconitic } & 2.22 & 66 & 334 \\ \text { Ascrbic } & 2.31 & 37 & 0 \\ \text { Citric } & 2.70 & 547 & 950 \\ \text { Crotonic } & 2.31 & 0 & - \\ \text { Diglycolic } & 2.30 & 0 & - \\ \text { Fumaric } & 1.90 & 407 & 949 \\ \text { Glutamic } & 5.85 & 551 & 138 \\ \text { Glycolic } & 2.32 & 0 & - \\ \text { Lactic } & 2.20 & 180 & 23 \\ \text { Levulinic } & 2.40 & \text { trace } & - \\ \text { Maleic } & 2.30 & 0 & - \\ \text { Malic } & 4.25 & 656 & 315 \\ \text { Malonic } & 2.30 & 0 & - \\ \text { Oxalic } & 2.88 & 138 & 0 \\ \text { Pyruvic } & 2.34 & 0 & - \\ \text { Succinic } & 1.94 & 654 & 897 \\ \text { Tartaric } & 6.60 & 328 & 485 \\ \text { Arabinose } & 1.72 & 545 & 1317 \\ \text { Glucose } & 1.62 & 610 & 826 \\ \text { Glycerol } & 1.97 & 596 & 964 \\ \text { Xylose } & 1.75 & 628 & 1250 \\ & & & \end{array}$

TABLE XXI

Effect of Organic Acids Added to Basal Medium $\left(25^{\circ}, 15\right.$ days $)$

\begin{tabular}{|c|c|c|c|}
\hline $\begin{array}{l}\text { Organic acid } \\
\qquad(1 \%)\end{array}$ & $\begin{array}{l}\text { Final } \\
\mathrm{pH}\end{array}$ & $\begin{array}{l}\text { Yield of } \\
\text { dry matter }\end{array}$ & $\begin{array}{l}\text { Total } \\
\text { carotene }\end{array}$ \\
\hline & & $\mathrm{mg} / 100 \mathrm{ml}$ & $\mu g / g d r y$ \\
\hline None & 1.71 & 582 & 808 \\
\hline Acetic & 2.30 & 0 & \\
\hline Adipic & 1.90 & 610 & 666 \\
\hline Butyric & 2.30 & 0 & — \\
\hline Citric & 2.62 & 716 & 660 \\
\hline Formic & 2.30 & 0 & - \\
\hline Fumaric & 1.90 & 429 & 1170 \\
\hline Glutaric & 1.87 & 688 & 712 \\
\hline$\alpha$-Ketoglutaric & 1.92 & 712 & 524 \\
\hline Kojic & 1.92 & 698 & 895 \\
\hline Lactic & 4.03 & 325 & 540 \\
\hline Levulinic & 1.70 & 122 & 230 \\
\hline Malic & 3.18 & 343 & 713 \\
\hline Dimethylacry- & 2.30 & 0 & - \\
\hline Mevalonic & 2.30 & 0 & - \\
\hline Oxalic & 5.49 & 516 & 270 \\
\hline Propionic & 2.30 & 0 & - \\
\hline Pyruvic & 2.30 & 0 & $\ldots$ \\
\hline Succinic & 1.90 & 674 & 684 \\
\hline Tartaric & 3.42 & 321 & 360 \\
\hline
\end{tabular}

their spectral characteristics and stereochemical properties.

Different solvents vary both the wavelength of maximum absorption and the absorbance of $\beta$-carotene. The former shows a correlation with the refractive index squared, and the latter a logarithmic correlation with wavelength. The solvent effects may be explained as dipolar interactions between $\beta$-carotene and the solvent.

Lycopene and $\beta$-carotene contain the same chromophore group, a series of 11 conjugated double bonds, but there are three distinguishing differences in their comparative spectral properties: (1) as already noted, the absorption for $\beta$-carotene are much less than for lycopene, (2) the wavelength of maximum absorption for $\beta$-carotene is displaced $21 \mathrm{~m} \mu$ hypochromically, and (3) the spectral fine structure characteristic of acyclic polyenes is reduced in the spectrum of $\beta$-carotene. These distinguishing properties can be explained on the basis of the unique properties of the $\beta$-ionone rings which terminate the conjugated double bonds in $\beta$-carotene. The spectral properties of several polyenes are listed in Table XXIII and the wavelengths of their maximum absorption in hexane are plotted against the number of conjugated double bonds in Fig. 3.

The effect one $\beta$-ionone ring in carotenes, e.g., $\alpha$ - and $\gamma$-carotenes, is to impart properties intermediate between $\beta$-carotene and the acyclic analogues. It is apparent that an $\boldsymbol{\alpha}$-ionone ring does not affect the chromophore of carotenoids because the double bond of $\boldsymbol{\alpha}$-ionone is not conjugated. A comparison of the spectral properties of known polyene related to the carotenoids shows that the wavelength of maximum absorptions is a function of the logarithm 
TABLE XXII

Effect of Several Organic Compounds $\left(25^{\circ}, 15\right.$ days $)$

\begin{tabular}{|c|c|c|c|c|c|c|c|}
\hline Compound & $\begin{array}{l}\text { Concen- } \\
\text { tration }\end{array}$ & $\begin{array}{l}\text { Yield of } \\
\text { dry matter }\end{array}$ & $\begin{array}{l}\text { Total } \\
\text { carotene }\end{array}$ & Compound & $\begin{array}{l}\text { Concen- } \\
\text { tration }\end{array}$ & $\begin{array}{l}\text { Yield of } \\
\text { dry matter }\end{array}$ & $\begin{array}{l}\text { Total } \\
\text { carotene }\end{array}$ \\
\hline Citral & $\begin{array}{c}m g / l \\
0 \\
0.1 \\
1 \\
10 \\
100\end{array}$ & $\begin{array}{c}m g / 100 m l \\
564 \\
550 \\
575 \\
553 \\
576\end{array}$ & $\begin{array}{c}\mu g / g \text { dry } \\
1052 \\
1129 \\
1096 \\
1132 \\
798\end{array}$ & Isophytol & $\begin{array}{c}m g / l \\
0 \\
0.1 \\
1 \\
0 \\
100\end{array}$ & $\begin{array}{c}m g / 100 m l \\
564 \\
565 \\
569 \\
569 \\
579\end{array}$ & $\begin{array}{c}\mu g / \boldsymbol{g} d r y \\
1054 \\
1429 \\
1061 \\
958 \\
1039\end{array}$ \\
\hline Citronellal & $\begin{array}{c}0 \\
0.1 \\
1 \\
10 \\
100\end{array}$ & $\begin{array}{l}552 \\
562 \\
552 \\
560 \\
554\end{array}$ & $\begin{array}{r}1039 \\
1020 \\
1050 \\
1029 \\
893\end{array}$ & Isoprene & $\begin{array}{r}0 \\
1 \\
10 \\
100 \\
1000\end{array}$ & $\begin{array}{l}555 \\
560 \\
554 \\
559 \\
556\end{array}$ & $\begin{array}{l}1098 \\
1066 \\
1034 \\
1088 \\
1105\end{array}$ \\
\hline Geraniol & $\begin{array}{c}0 \\
0.1 \\
1 \\
10 \\
100\end{array}$ & $\begin{array}{l}545 \\
578 \\
576 \\
564 \\
504\end{array}$ & $\begin{array}{r}1075 \\
1021 \\
955 \\
971 \\
982\end{array}$ & $\begin{array}{l}\text { Methyl } \\
\text { heptenone }\end{array}$ & $\begin{array}{r}0 \\
1 \\
10 \\
100 \\
1000\end{array}$ & $\begin{array}{r}548 \\
562 \\
565 \\
521 \\
0\end{array}$ & $\begin{array}{l}1077 \\
1060 \\
1071 \\
1265\end{array}$ \\
\hline$\alpha$-Ionone & $\begin{array}{c}0 \\
0.1 \\
1 \\
10 \\
100\end{array}$ & $\begin{array}{l}574 \\
582 \\
566 \\
569 \\
498\end{array}$ & $\begin{array}{r}1037 \\
1067 \\
1120 \\
1312 \\
954\end{array}$ & Phytol & $\begin{array}{l}0 \\
0.1 \\
1 \\
10 \\
100\end{array}$ & $\begin{array}{l}562 \\
572 \\
581 \\
579 \\
575\end{array}$ & $\begin{array}{r}1029 \\
1204 \\
1085 \\
966 \\
1041\end{array}$ \\
\hline$\beta$-Ionone & $\begin{array}{c}0 \\
0.1 \\
1 \\
10 \\
100\end{array}$ & $\begin{array}{l}568 \\
564 \\
557 \\
552 \\
489\end{array}$ & $\begin{array}{r}1034 \\
1234 \\
1255 \\
1450 \\
894\end{array}$ & Squalene & $\begin{array}{c}0 \\
0.1 \\
1 \\
10 \\
100\end{array}$ & $\begin{array}{l}559 \\
533 \\
562 \\
529 \\
538\end{array}$ & $\begin{array}{r}1070 \\
1053 \\
959 \\
1209 \\
1061\end{array}$ \\
\hline$\psi$-Ionone & $\begin{array}{r}0 \\
1 \\
10 \\
100 \\
1000\end{array}$ & $\begin{array}{r}568 \\
579 \\
580 \\
475 \\
0\end{array}$ & $\begin{array}{r}1088 \\
1134 \\
1306 \\
904 \\
-\end{array}$ & Vitamin A & $\begin{array}{r}0 \\
1 \\
10 \\
100 \\
1000\end{array}$ & $\begin{array}{l}548 \\
620 \\
631 \\
502 \\
165\end{array}$ & $\begin{array}{l}1058 \\
1044 \\
1009 \\
1257 \\
1302\end{array}$ \\
\hline
\end{tabular}

TABLE XXIII

Absorption of Carotenes and Polyenes in Hexane

\begin{tabular}{|c|c|c|c|c|c|c|}
\hline Polyene & $\lambda_{\max }$ & $A$ & $\varepsilon \times 10^{3}$ & $N$ & $\mathrm{Ne}$ & Ref. \\
\hline & $m \mu$ & & & & & \\
\hline Lycopene & 472 & 348 & 186 & 11 & 11 & (8) \\
\hline $\boldsymbol{\gamma}$-Carotene & 462 & 272 & 146 & 11 & 10.3 & (8) \\
\hline$\delta$-Carotene & 456 & 320 & 172 & 10 & 10 & (9) \\
\hline$\beta$-Carotene & 451 & 256 & 137 & 11 & 9.6 & \\
\hline$\alpha$-Carotene & 446 & 272 & 146 & 10 & 9.3 & $(10)$ \\
\hline Neurosporene & 439 & 299 & 161 & 9 & 9 & (11) \\
\hline$\zeta$-Carotene & 400 & 220 & 119 & 7 & 7 & (12) \\
\hline Phytofluene & 348 & 152 & 82 & 5 & 5 & (13) \\
\hline Phytoene & 286 & 85 & 46 & 3 & 3.3 & (14) \\
\hline$\alpha$-Eleostearic acid & 272 & 170 & 47 & 3 & 3 & (15) \\
\hline
\end{tabular}

$A$, absorbance $(\mathrm{mg} / \mathrm{ml})$

$\varepsilon$, molar absorbance (moles/liter)

$N$, number of conjugated double bonds

$\mathrm{Ne}$, apparent number of conjugated double bonds 


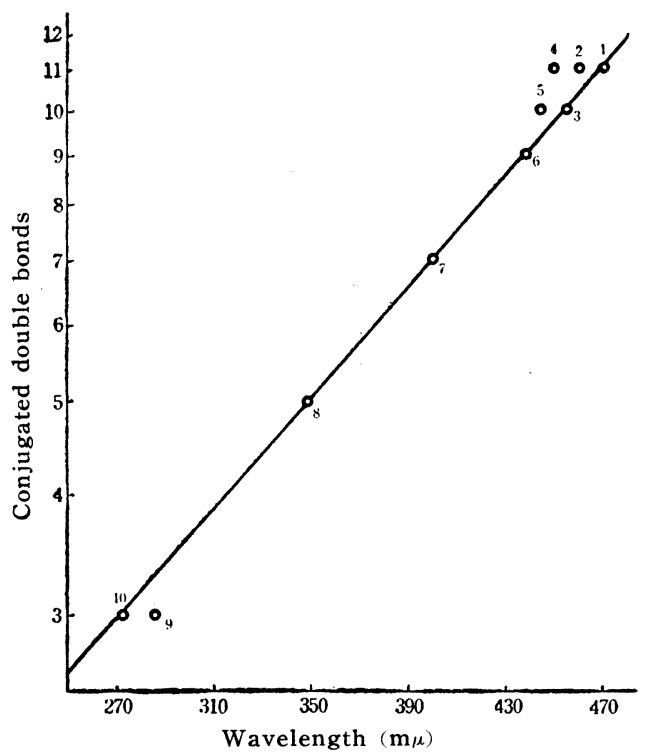

Fig. 3 Relationship between Maximum Absorption of Polyenes and Number of Conjugated Double Bonds of number of conjugated double bonds. Extrapolation of the wavelengths of maximum absorption of those carotenes containing $\beta$-ionone rings suggests that each $\beta$-ionone ring contributes in effect only 0.3 of a double bond to the conjugated chromophore. It could be explained that the $\beta$-carotene molecule is capable of undergoing transition only when the conjugated double bonds are coplanar. There is a steric hindrance to coplanarity between the 2 methyl groups at $C_{1}$ and the hydrogen at $\mathrm{C}_{8}$ in $\beta$-carotene molecule.

This steric overlap cannot be relieved by bending $\mathrm{C}-\mathrm{C}$ bond at $\mathrm{C}_{6}$ because of the proximity of the hydrogen at $\mathrm{C}_{7}$ to the methyl group at $\mathrm{C}_{5}$. Since the steric overlap cannot be relieved by bending the bond angles, the plane of the $\beta$-ionone ring takes a position angular to the plane

of the conjugated double bonds. In so far as the two planes are transangular, maximum resonance or maximum orbital overlap cannot be achieved, and the electronic transition is forbidden.

The concept of steric hindrance to a coplanar arrangement with its consequent effects on molecular spectra is well established (16). The fact that allene and ketene do not show a K-band above $200 \mathrm{~m} \mu$ (17) because the $\pi$-electron orbitals are at right angles to each other, lends support to the limited contribution of the $\beta$-ionone double bond to the conjugated system in $\beta$-carotene.

The transangular nature of $\beta$-ionone ring may posibly offer an explanation for its unique ability to undergo a kind of selective reduction in carotenes (18).

\section{Biosynthetic Pathway of Carotenoids}

Succinic acid-1-C ${ }^{14}$ was added to the culture medium of Pen. sclerotiorum containing succinic acid as the sole carbon source, and the incubation was continued. Pigment separation and measurement of the radioactivity were made 15 days after inoculation. The activity of $\beta$-carotene was pretty low in 15 days, but much increased in 20 days. The activity of $\boldsymbol{\gamma}$-carotene was also increased in 20 days. The pathway of carotenoid formation was discussed as follows :

Conceptual possibilities and pathways for the phytosynthesis of carotenoids were stimulated by the Porter-Lincoln theory of carotenogenesis in the ripening tomato (19). Theories of carotene formation have been reviewed with a detailed consideration given to the Porter-Lincon concept of a series of quadridehydrogenation steps (20). If the dehydrogenation pathway is taken into 
consideration as a provisional hypothesis, the relevant evidence and observations should possibly be composed into a unified rationale which may be tested by investigators in related disciplines. The implications inherent in a coordinated rationale of more recent evidence bearing on carotene biosynthesis merits candid consideration.

Chemical studies on the acyclic polyene (Porter-Lincoln) series have indicated that all are isoprenic and bear an isologous relationship to lycopene. Chemical dehydrogenation of the polyenes, phytoene and phytofluene, into the more unsaturated members of the acyclic polyene series (21), and the wellknown oxygen requirement for carotenoid formation in plants and microorganisms lend some support to the phyto-dehydrogenation mechanism as a general possibility. As a rationalization $\beta$-carotene may be deemed the objective of carotene phytosynthesis; however, $\beta$-carotene is not necessarily constrained to a static state. It apparently exists in a dynamic state in green leaves (22) and may be catabolized via its epoxide and furanoid (23).

Phytoene bears a structural similarity to squalene which is usually associated with animal metabolism. All evidence, including infrared evidence, points to a definite quasi-cyclic structure for squalene with the double bonds in a trans configuration $(24,25)$. In this configuration, squalene is cyclized by a concerted, cationic, non-stop reaction (26) to lanosterol (27). Phytoene has strong infrared absorption bands at 6.1 and $12.9 \mu$ (14) which indicates that the central double bond of the triene conjugation in phytoene is cis (28). Otherwise the infrared absorption spectrum is identical to that of squalene bearing out its structural relationship to squalene. If phytoene does not function as a carotene precursor, it is interesting to consider its possible role as a precursor to plant steroids and triterpenes or as a reservoir of metabolic "isoprene."

$\eta$-Carotene, which is possibly a cyclized analog of $\zeta$-carotene $(29,30)$ has been found among plant carotenoids and probably exists in tomatoes and carrots. It is proposed for purposes' of this discussion that cyclization occurs with $\zeta$-carotene to form $\eta$-carotene.

There are a number of reasons militating against considering the cyclization of lycopene to form $\beta$-carotene (20). Among them are the greater thermodynamic stability of lycopene and the overt steric hindrance to direct cyclizations indicated previously. These same arguments apply in part to a consideration of neurosporene as an immediate precursor to $\beta$-carotene. If cyclization were to occur at the phytoene level, a steroid-like molecule could very well result from a concerted, non-stop reaction.

In order to unify the known polyenes and carotenes into a rational synthetic relationship, phytoene is considered to be the initial member arising from fundamental proceses of "isoprene" synthesis. According to the proposed concept, bis-dehydrogenation first produces phytofluene and then $\zeta$-carotene which is deemed the pivotal polyene. On the one hand, cyclization of $\zeta$-carotene may lead to $\eta$-carotene, quadri-dehydrogenation of $\eta$-carotene to $\varepsilon$-carotene, and isomerization of $\varepsilon$-carotene to $\alpha$-carotene and then to $\beta$-carotene. On the other hand, bis-dehydrogenation of $\zeta$-carotene leads to neurosporene which in 
turn may be dehydrogenated to lycopene, or which by sequential cyclization, dehydrogenation, and isomerization may lead to $\delta$ - and then to $\gamma$-carotene.

The remarkable observations of Claes on mutants of Chlorella vulgaris seem to substantiate this dehydrogenation concept and confirm its application to chlorophyllous tissue (31). One mutant (5/871) accumulated phytoene and another accumulated phytoene, phytofluene, and $\zeta$-carotene. This observation can be explained by a post-phytoene dehydrogenation block and post- $\zeta$-carotene cyclization block, respectively. There were no mutants which accumulated phytofluene exclusively or both phytoene and phytofluene possibly because the same reaction (perhaps the same enzyme) dehydrogenating phytoene to phytofluene also forms $\zeta$-carotene. Zeaxanthin and lutein (xanthophyll) were blocked in the mutants which produced phytoene, phytofluene, and $\zeta$-carotene; however, one mutant $(9 \mathrm{a})$ produced xanthophyll, but no $\alpha$ - or $\beta$-carotene. This observation suggests that the phytosynthesis of carotenes and xanthophylls diverge at some level subsequent to the synthesis of $\zeta$-carotene. A logical explanation would be that oxygen is introduced in position 3 of the carotene at the $\eta$ - or $\varepsilon$-carotene level. From this point the syntheses of carotenes and xanthophylls could follow parallel pathways.

\section{SUMMARY}

1. In addition to the strains known as carotene producers, Pen. sclerotiorum, Pen. multicolor, Pen. lapidosum, and Rhizoctonia zeae were found to be new carotene producers, and carotenoid pigments produced by these fungus strains were identified and determined.

2. Pen. sclerotiorum NRRL 2074, the best carotene producer, was investigated on its culture condition in some detail, and its carotene content was enhanced up to $1.85 \mathrm{mg}$ total carotene per gram dry mat. Particularly, low $\mathrm{pH}$ value of culture medium ( $\mathrm{pH}=2 \pm 0.25)$, and addition of nitrogen-containing methyl donor, e.g., betaine and choline, into the medium, were found to stimulate its carotene production remarkably.

3. The stereochemical and spectral properties of the carotenoid and its biosynthetic pathway were discussed.

\section{ACKNOWLEDGMENT}

These investigations were carried out in part during the author's stay in the Department of Biochemistry, Purdue University, U.S.A. Grateful acknowledgment is expressed to Dr. Ryohei Takata in Kyoto University, and Dr. F. W. Quackenbush and Dr. W. J. Rabourn in Purdue University for their guidance and encouragement during the course of this study.

\section{REFERENCES}

1. Lutheraan, P. J., and Choay, J., Ann. Parasitol. Humaine et Comparée 22, 89 (1947).

2. Mase, Y., Rabourn, W. J., and Quackenbush, F. W., Arch. Biochem. Biophys. 68, 150 (1957). 
3. Raper, K.B., and Thom, C., A Manual of the Penicillia. Williams and Wilkins Co., Baltimore, Md. (1949).

4. Jackson, R. W., Personal Communication.

5. Curtis, T.P., and Reilly, J., Biochem. J. 34, 1419 (1940).

6. Birkinshaw, J.H., ibid. 52, 283 (1952).

7. Watanabe, H., J. Pharm. Soc. Japan 72, 807 (1952).

8. Zechmeister, L., LeRosen, A.L., Schroeder, W. A., Polgar, A., and Pauling, L., J. Am. Chem. Soc. 65, 1940 (1943).

9. Porter, J. W., and Murphy, M. M., Arch. Biochem. Biophys. 32, 21 (1951).

10. Zscheile, F.P., White, J. W., Beedle, B. W., and Reach, J.R., Plant Physiol. 17, 331 (1942).

11. Haxo, F., Arch. Biochem. 20, 400 (1949).

12. Portor, J. W., Nash, H. A., and Zscheile, F. P., ibid. 7, 305 (1945).

13. Koe, B. K., and Zechmeister, L., Arch. Biochem. Biophys. 46, 100 (1953).

14. Rabourn, W. J., and Quackenbush, F. W., ibid. 48, 267 (1954).

15. Herb, S. F., and Riemenschneider, R. W., J. Am. Oil Chem. Soc. 29, 456 (1952).

16. Jones, R. N., J. Am. Chem. Soc. 65, 1818 (1943).

17. Braude, E. A., Determination of Organic Structure by Physical Methods, p. 147 Academic Press, New York (1955).

18. Karrer, P., and Ruegger, A., Helv. Chim. Acta. 23, 955 (1940).

19. Porter, J. W., and Lincoln, R. E., Arch. Biochem. 27, 390 (1950).

20. Goodwin, T.W., Carotenoids, Their Comparative Biochemistry, p. 63. Chemical Publishing Co., Inc., New York (1954).

21. Zechmeister, L., and Koe, B. K., J. Am. Chem. Soc. 76, 2923 (1954).

22. Bandurski, R.S., Bot. Gaz. 111, 95 (1949).

23. Glover, J., and Redfern, E. R., Biochem. J. 54, viii (1953).

24. Woodward, R. B., and Block, K., J. Am. Chem. Soc. 75, 2023 (1953).

25. Nicolaides, N., and Laves, F., ibid. 76, 2596 (1954).

26. Eschenmoser, A., Ruzicka, L., Jeger, O., and Arigoni, D., Helv. Chim. Acta 38, 1890 (1955).

27. Chen, T. T., and Block, K., J. Am. Chem. Soc. 78, 1516 (1956).

28. Lunde, K., and Zechmeister, L., ibid. 77, 1647 (1955).

29. Goodwin, T. W., Biochem. J. 51, 458.(1952).

30. Joyce, A. E., Nature 173, 311 (1954).

31. Claes, H., Z. Naturforsch. 9b, 461 (1954). 